\title{
Maximizing HIV partner notification opportunities for index patients and their sexual partners in Malawi
}

\section{G Kamanga ${ }^{1}$, L Brown ${ }^{2}$, P Jawati ${ }^{1}$ \\ D Chiwanda ${ }^{1}$, N Nyirenda ${ }^{1}$}

1. UNC Project-Malawi, Lilongwe, Malawi

2. Department of Epidemiology, University of North Carolina, Chapel Hill, North Carolina, USA

\begin{abstract}
\section{Introduction}

HIV testing and counselling (HTC) is important to effect positive sexual behaviour change and is an entry point to treatment, care, and psychosocial support. One of the most practical initiatives to increase HTC is to encourage sexual partners of HIV-infected persons to test for HIV. However, partner notification strategies must be feasible in the healthcare setting and acceptable to the population.
\end{abstract}

\section{Methods}

We conducted a qualitative study during the pilot phase of an HIV partner notification trial to complement its assessment of feasibility and acceptability of methods of partner notification. We performed in-depth interviews with 16 consecutive HIV-positive index participants who consented and their 12 identifiable sexual partners. We also conducted two focus group discussions with healthcare workers to supplement the patient perspectives.

In the main study, newly diagnosed HIV cases (index cases) were randomized to one of three methods of partner notification: passive, contract, and provider referral. Clients in the passive referral group were responsible for notifying their sexual partners themselves. Individuals in the contract referral group were given seven days to notify their partners, after which a healthcare provider contacted partners who had not reported for counselling and testing. In the provider group, a healthcare provider notified partners directly.

\section{Results}

Although most index participants and partners expressed a preference for passive notification, they also highlighted benefits for provider-assisted notification and the universal right for all HIV-exposed persons to know their HIV exposure and benefit from HIV testing and access antiretroviral treatment. Several participants mentioned couples counselling as a way to diffuse tension and get accurate information. All mentioned benefits to HIV testing, including the opportunity to change behaviour.

\section{Conclusions}

Provider-assisted partner notification is not preferred, but it is acceptable and may complement the passive method of notification. Couples counselling should also be encouraged.

\section{Introduction}

Partner notification is the process of informing the sexual contacts of an individual who has a sexually transmitted infection (STI), such as HIV, of their possible exposure and encouraging them to seek counselling and testing themselves. The objectives of partner notification include providing earlier diagnosis and treatment for the partners of infected individuals, with aims of reducing onward transmission, preventing consequences of undiagnosed infections, and providing an opportunity to discuss safer sexual behaviour with sexual partners ${ }^{1}$.

Despite the large burden of HIV/AIDS in Africa, many people remain unaware that they are HIV-positive and disclosure rates are low ${ }^{2}$. Fear of rejection or abandonment, especially by a main partner, is commonly cited as the main reason why HIV-positive individuals avoid partner notification ${ }^{3,4}$. Inability to trace a partner, especially casual partners, is also associated with failure to disclose ${ }^{3}$. If disclosure does occur, apprehension about the consequences of disclosure often causes hesitancy and delayed disclosure, which is a barrier to engaging in preventive behaviour ${ }^{5}$. Effective partner notification programmes can help increase disclosure to sexual partners and encourage HIV testing among the sexual partners of those infected with $\mathrm{HIV}^{6}$.

The traditional method of partner notification for STIs, including HIV, in Malawi is passive referral, which involves the index patient notifying his or her sexual partners about HIV exposure or status. In Malawi, less than one-third of sexual partners present for STI and HIV testing and counselling services after being notified through passive referral ${ }^{7}$. Partner notification methods that involve healthcare providers assisting index cases to disclose have increased uptake of sexual partners receiving STI treatment ${ }^{3}$.

There is a need to explore more about the best partner notification strategies and methods for HIV disclosure in the sub-Saharan region ${ }^{8}$. Provider-assisted referral has led to increased HIV testing among sexual partners of newly diagnosed HIV cases in developed countries?

In our main trial on the feasibility and effectiveness of partner notification in Malawi, the assessment of uptake of the different methods of partner notification showed the following proportions of partner return: passive (whereby the onus of partner notification is entirely on the index patient) 24\%, contract (index patient is given a set time limit to notify partners, after which the provider will notify them) $51 \%$, and provider notification $51 \%{ }^{10}$. This demonstrated the effectiveness of provider-assisted partner notification for STIs and HIV. Data from a large programme implementation in Cameroon showed that provider-assisted partner notification not only yielded high proportion of partners $(83.8 \%)$ seeking counselling and testing, but also that the majority of partners favoured the approach ${ }^{11}$.

Provider-assisted partner notification requires additional care providers for implementation, which is difficult in resourcelimited setting. However, cost-effectiveness analysis of our main trial showed that provider-assisted partner notification is reasonably cost-effective in terms of dollars per HIV transmission averted. In a cohort of $5000 \mathrm{HIV}$-positive indexes, it was estimated that 27.5 and 27.9 transmissions would be averted over one year with contract and provider notification, respectively, compared to passive notification, and that the cost per partner tested were $\$ 9, \$ 19$, and $\$ 4$, respectively ${ }^{12}$.

The relative cost implication of provider partner notification calls for targeting the approach to those who require the service. Our previous work demonstrated that the following characteristics and contexts benefited from provider partner notification: male partners, non-main partners, relationship duration of less than 24 months, STI other than genital ulcer disease diagnosed in index patient, and greater than primary education in the index. To achieve success for effective partner notification, both index patients' and sexual partners' opinions about these methods need to be sought ${ }^{13}$. It is important to get wider perspectives about the acceptance of these strategies. We sought to get insights using in-depth 
interviews with index patients and their sexual partners, which were done as part of our main partner notification study.

\section{Methods}

We conducted qualitative study during the pilot phase of an HIV partner notification trial between 31 October 2008 and 30 January 2009 at Kamuzu Central Hospital STI Clinic in Lilongwe, Malawi. In the main clinic trial, we randomized 240 patients with newly diagnosed HIV and other STIs to one of three methods of partner notification: passive referral $(\mathrm{n}=77$ ), in which index patients were asked to inform their sexual partner(s); contract referral $(n=82)$, in which index patients were asked to inform their sexual partner(s) and, if partners did not report to clinic within seven days, then a healthcare worker would help inform the partners; and provider referral $(\mathrm{n}=81)$, in which the provider inform the sexual partner(s) within 48 hours ${ }^{10}$. In all arms, index cases were encouraged to immediately inform their sexual partners and were provided notification cards.

We invited consecutive index patients and their sexual partners who received HIV partner notification services to complete in-depth interviews about the partner notification process; 16 in-depth interviews were with index participants, of which 10 were females. There were six index interviewees from the passive referral group, five from the contract referral group, and five from the provider referral group. We completed 12 in-depth interviews with sexual partners, of which seven were females. There were three partner interviewees from the passive referral group, two from the contract referral group, and seven from the provider referral group. Since this kind of qualitative enquiry involved detailed information from participants, the selected samples were adequate to reach saturation.

We also sought the views of healthcare workers through two focus group discussions. The first focus group had nine participants and the second focus group had five participants. The research staff were exempted from the discussions. The interviews and focus group discussions lasted between 45 minutes and one hour.

All interviews were conducted in the local language (Chichewa) by trained social scientists and were audiotaped. Interviews were transcribed and then translated into English. Data were manually categorized in tables according to participant type. The codes and themes identified matched the predetermined ones that were based on the study objectives and in-depth questionnaire guides. The derivation of themes was guided by the literature review, field experience, and real-time evaluation of the data. Transcripts were read and reread and no emerging themes were identified. We manually analyzed the data according to the coded data described above. Ethical approval of the study was obtained from the National Health Sciences Research Committee and the University of North Carolina Institutional Review Board.

\section{Results}

Most of the index participants who disclosed and all four who did not disclose indicated that disclosure or notification is important, as it is a way to link to care. Participants gave various experiences and insights regarding disclosure of HIV test results to their sexual partners.

\section{Preferred methods of partner notification}

Passive notification was the preferred method of disclosure among index patients and their partners. However, it is important to weigh situations on a case-by-case basis and then come up with the best partner notification approach. Some men and women do not inform their sexual partners if infected with HIV. For women, failure to disclose often resulted from fear of their male partner's reactions. A female index participant who disclosed through the passive approach (even though she was assigned to the provider referral arm) said:

"Firstly, you are supposed to know how your partner's behaviour is, because others are very difficult, and the moment you tell them that you have been diagnosed with HIV, you will be in trouble. So if you know that you will definitely bring chaos in the home if you told your spouse yourself, it's better to ask the clinic personnel to do it for you. But if your husband is very understanding, then you can tell him yourself, like how I did with my partner."

This was corroborated by another female index participant who was assigned to the passive arm and disclosed as such:

"The best way is like what has happened to us, me and my husband. First I came here and got tested and I was told about my test results. Then I also went to notify my partner. I have my own way of notifying him as my husband, and I know how I can approach him."

Another important method of disclosure of HIV testing results is couple's counselling. A male index participant who was assigned to the contract arm and did not disclose his HIV test result stated:

"The best way is just to tell her that, let's go to the clinic to get tested and receive special counselling (couple's counseling)."

Although most participants preferred passive notification, many also supported provider-assisted notification. A male partner who was informed through passive notification mentioned and had no problem with provider-assisted notification said:

"... if I had met this health worker I could still have come to the clinic for a test just the same as how I have been told by my wife."

Many female partners also echoed their preference of provider-assisted partner notification. Here is of what two women said in support of this:

"Ah, no, I liked this way (provider notification) very much because if they had told him to inform his partner maybe he could not have told me about it."

"If you feel that your partner will chase you or end the relationship you can tell the clinic staff that my husband is stubborn and you bave to give me a notification slip or tell me another method of me bringing my partner here so that you can tell us together about our HIV status." 
Table 1: Preferred methods of partner notification among the index participants who disclosed to their partners

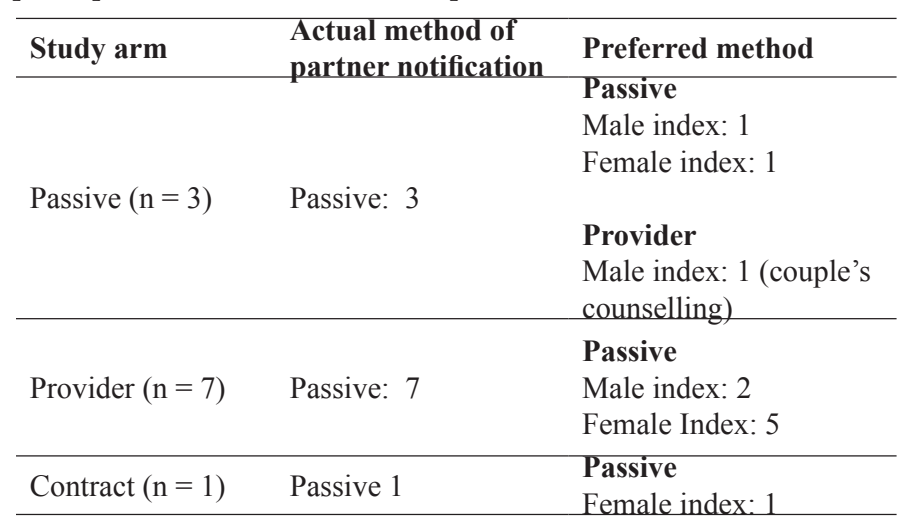

Most health care workers supported passive notification as the best method but indicated that couple's counseling needed to be encouraged. However, they also reiterated that it is not easy to disclose one's HIV results to sexual partners and notification depends on the type of partner. A healthcare worker, in a focus group discussion, said:

"... have enrolled 35 acute HIV clients but only twelve partners of these have been notified, which shows that there is a big problem with disclosure of HIV infection to partners. But when we also look where most of these got the infection from they tell us that it was just a "bit and run' and there is no way they could go back to them with a notification."

\section{Importance of testing after being notified}

Healthcare workers, index participants, and partners all agreed that HIV testing after being notified is important because it facilitates positive behavior change, entry to early medical care, prevention of spread of HIV to the notified partner (in discordant couples), and prevention of re-infection. A female partner stated the importance of HIV testing:

"You know how you can protect yourself or, if you are a discordant couple, you know how you can protect your sexual partner so that other one should not be infected."

The importance HIV testing after being notified was reiterated by a healthcare provider during a focus group discussion:

"I think... has said it all. But maybe just to add on some of the benefits, apart from the issues to do with change in behavior-I think a partner who has been informed about a positive HIV result should consider it as a blessing in the sense that this partner is going to have an opportunity of accessing medical care very early..."

Similarly, participants indicated that notification would help employ measures to protect each other against infection. A male partner indicated that if one does not know his or her status, he or she will continue with promiscuous behaviour and continue spreading HIV to more people.

\section{Gender implications}

Women are victims of gender discrimination related to HIV disclosure as one of the participants of a focus group discussion indicated:

"Mostly we see that it is the women who come for testing that find it difficult to disclose to their male partners compared to male clients. This is so because, in the majority of families here, men are the breadwinners and therefore their wives have very little authority over what happens in the home; they always wait for the man to make all the decisions."

There was an indication from the findings that women depend on their husband's or sexual partner's permission to seek medical services, as a female index patient explained:

"... That's a problem, and I am worried with that. He will ask me why I came to hospital without telling him, that's the problem."

The issue of seeking permission from one's male partner before attending health care was corroborated by another female index participant:

"He did not scold me in any way because when I was coming here be gave me permission to come for an HIV test, and he knew that I have come here for HIV testing. "

\section{Factors influencing disclosure}

Some participants cited advantages of partner notification, especially passive partner notification, because it promotes confidentiality and builds trust among partners. One focus group discussion participant said:

"I feel this brings trust to the partner because if you have disclosed to your partner whether you are positive or negative they will understand you, other than hearing it from a third party it is more painful and brings in anger."

The other factor mentioned by almost all participant groups in favour of partner notification was that it is an opportunity for both partners to know their health status, which would result getting HIV/AIDS-related care together.

Blame shifting and ventilation of anger was another interesting reason given for partner notification. One of the healthcare workers mentioned the following during a focus group discussion:

"Some choose to tell their partners because they want to shift the blame for their infection (in order to get psychological relief). Sometime back, a female client told me that she had been warning her husband about his promiscuity and womanizing. When she tested HIV positive she told me she would confront her husband for being responsible for this due his waywardness. To her it was obvious that it was her husband who infected her."

\section{Social harms related to partner notification}

Social harms related to disclosure of HIV status, especially marital or relationship breakdowns, were feared by both healthcare providers and participants. The fears were more pronounced among the healthcare workers than participants. Concerns about social harms were greater among women, especially if infected. Some female index participants did not disclose because of fear of negative repercussions from their male partners. The sentiments of two female index participants were as follows:

"He would just have abandoned me. The relationship would have ended there.... The marriage would end there and then. He is someone who does not take time to hear what the other person has to say."

\section{"I am afraid... Yes, he will divorce me [if I disclose to him]."}

Despite concerns about social harms, there were no social harms reported by any study participant as a result of disclosure in this study.

\section{Discussion}

http://dx.doi.org/10.4314/mmj.v27i4.5 
The findings of this qualitative study have shown that the passive method is the most preferred method of partner notification by index patients, their sexual partners, and healthcare providers. Many participants also indicated that provider-assisted partner notification could potentially help clients with disclosure in relationships that would likely be under after (self-)disclosure of HIV status. This means, depending on the situation, provider-assisted partner notification and couple's counselling complement passive notification. This conditional view by participants is supported by the findings of our main trial, which demonstrated the effectiveness of provider-assisted partner notification interventions ${ }^{10}$.

The cost-effectiveness analysis of the main trial, and the estimated 27.9 new HIV infections per 5000 index patients that would be averted with contract and provider notification $^{12}$ point the potential benefits of implementing provider-assisted HIV status disclosure in Malawi. In this study, partner tracing was done by a cadre of healthcare workers equivalent to existing health surveillance assistants ${ }^{10}$. We think this approach could easily be implemented outside the trial setting because there are already many trained health surveillance assistants in Malawi who could implement this.

The in-depth interviews revealed that although participants found it socially desirable and preferable to disclose HIV testing results to their partners themselves, fear of their partners' reactions made it acceptable to have a health care worker assist them with disclosure. This finding is in line with the World Health Organization's recommendation that passive referral should be a starting point for partner notification and that provider-assisted referral should be used only to complement it $^{14}$.

In a different study but similar setting, participants knew the importance of disclosure or partner notification, but the reason for failure to disclose was commonly the false assumption they held that if a person is infected with HIV then their sexual partners must surely be infected as well ${ }^{15}$.

While our main trial showed that more sexual partners receive counselling and testing when provider-assisted methods were used $^{10}$, this qualitative study gave a real and wider perspective on the partner notification options.

The understanding of sexual partners' behaviours and anticipated reactions also came out as a justification to use passive notification. This is in line with findings in Uganda, where it was observed that psychosocial factors, such as a positive attitude and previous success in having referred a partner influenced participants to notify their partners themselves ${ }^{16}$. Although the preference was passive notification as opposed to provider notification, our main study still showed good uptake of provider partner notification (51\%) compared to the standard of care passive notification $(24 \%)^{10}$. It is therefore evident that, even though people prefer the passive method, the lack of confidence to approach their sexual partners, brought about by fears of broken relationships or gender-related barriers, often prevents people following through with self-disclosure. From such findings we can envisage that setting up purposeful providerassisted partner notification programmes would be helpful to increase disclosure in the long term. A large programme setting in Cameroon has shown that such a programme can be implemented with negligible social harms ${ }^{11}$. The programme in Cameroon provided a huge positive health impact, in the form of increased access to care and support.
Like our trial, provider notification was effective, but, in contrast to our work, they found the provider approach was the preferred method among participants ${ }^{11}$. It is possible that our observed preference of passive notification may change with increased implementation of such programmes, especially when social harms are minimal and when the benefits of care and support become more obvious to people.

Our study hinted that gender dynamics was one of the issues affecting partner notification. Some female index participants expressed reluctance to discuss partner notification issues with their partners to avoid negative reactions, because they did not inform their husbands in advance of their medical visits. Concerns about social harms were greater if it was the woman who was the index patient. Consequently, some female index participants did not disclose because of fear of negative repercussions from their male partners. This validates findings from our analysis of predictors of partners not reporting after being notified, which highlighted male gender as one such factor ${ }^{13}$.

In our setting some cultures confer more authority and influence on the man. This extends to health seeking practice. Anecdotal reports from clinical practice in Malawi indicate that some cultures require that women seek their husbands' permission to attend medical services. Likewise a man is "culturally forgiven" if he is found to be the source of an STI or HIV infection, but if a woman is found to be the source (sadly, sometimes she may just be simply the exhibitor of the infection the very same man may have contracted elsewhere), there is no cultural "backing for her behaviour". This cultural practice and gender marginalization creates a lot of fear and uncertainty for women to disclose, and this is a great hindrance to open communication regarding partner notification.

\section{Limitations and challenges}

The sexual partners are not necessarily the people with primary problem seeking the care or testing. In our setting sexual partners are invited to report to clinical care settings that are generally not well-equipped to provide confidential and timely services, because of inadequate personnel and infrastructure. This may have made some partners not report to the designated clinics.

Another challenge is that our interviews did not include partners who did not show up to the clinic. The reasons for such a problem could have been sexual encounters with unknown partners, particularly with sex workers or partners with homes that are difficult to locate ${ }^{18}$. The absent partners' preferences and perspectives could have enriched our results about the best method of partner notification.

It is good that social harms and stigma and discrimination were not evident in this study. However, our study did not have long-term follow-up. Therefore, the presence of social harms may have been underestimated.

This study was done in an STI clinic setting, and this might have influenced partners reporting because the stigma associated with STI clinics may have caused some partners to be reluctant to report. We, therefore, may have captured fewer partners than if the study was done in a more neutral setup. Since the study was connected with STI patients, the presence of symptoms may have enhanced partner return to the clinic. This is supported by our analysis of predictors for non-reporting to clinic, which showed that participants 
reported more often if the index had "a bothersome" STI (for example, genital ulcer) ${ }^{13}$. Some partners may not have reported to our public clinic because of perceived low care or status of most public health facilities. An alternative plan of linkage with private clinics was a missed opportunity to get a diverse partner representation.

Refusers were not captured in this phase; therefore, we may have missed people with other strong views about partner notification. However, only one tenth of the eligible participants refused participation in the main study.10 With this trend we most likely captured the major perspectives of the participants.

Regardless of the limitations, to our knowledge, this study presents a great opportunity to test partner notification methods for both HIV and other STIs in Malawi and other similar settings.

\section{Conclusions}

Effective partner notification is valuable, as it can bring about a multiplying effect on the uptake of HIV services and access to related care. The findings of our qualitative interviews show that most participants preferred passive notification and yet our main trial ${ }^{10}$ showed that provider-assisted partner notification is feasible and acceptable. The participants also reiterated the importance of engaging the help of healthcare workers when a client is not confident enough to disclose to his or her sexual partner. We recommend that HIV/AIDS and STI policies and programmes facilitate the implementation of provider-assisted partner notification (contract referral) to complement the traditional passive notification. Our study depended on partners reporting to clinics, but there is need for a new research opportunity to ascertain uptake and acceptability of partner notification whereby healthcare workers approach participants in their homes. This will help access partners who are locatable but not willing to report to health facilities for various reasons.

\section{References}

1. Ward H. Partner notification and contact-tracing. Medicine (Elsevier). 2005 Sep 1;33(9):28-30. doi:10.1383/medc.2005.33.9.28.

2. Bond VA. "It is not an easy decision on HIV, especially in Zambia": opting for silence, limited disclosure and implicit understanding to retain a wider identity. AIDS Care. 2010;22 Suppl 1:6-13. doi: 10.1080/09540121003720994.

3. Clark JL, Long CM, Giron JM, Cuadros JA, Caceres CF, Coates TJ, et al.; NIMH Collaborative HIV/STD Prevention Trial. Partner notification for sexually transmitted diseases in Peru: knowledge, attitudes, and practices in a high-risk community. Sex Transm Dis. 2007 May;34(5):309-13.

4. Bobrow EA. Factors that influence disclosure and program participation among pregnant HIV-positive women: a mixed methods study in Lilongwe, Malawi [dissertation]. Chapel Hill (NC): University of North Carolina at Chapel Hill; 2008.

5. Deribe K, Woldemichael K, Wondafrash M, Haile A, Amberbir A.Disclosure experience and associated factors among HIV positive men and women clinical service users in Southwest Ethiopia. BMC Public Health. 2008 Feb 29;8:81. doi: 10.1186/1471-2458-8-81.

6. King R, Katuntu D, Lifshay J, Packel L, Batamwita R, Nakayiwa S, et al. Processes and outcomes of HIV serostatus disclosure to sexual partners among people living with HIV in Uganda. AIDS Behav. 2008 Mar;12(2):232-43.
7. Kornfield R, Chilongozi D. Health seeking behaviour and partner notification by clients with sexually transmitted diseases (STDs) in Malawi: an ethnographic approach. Lilongwe: John Snow, Inc.-Support to AIDS and Family Health (JSI-STAFH) Project; 1997.

8. Mathews C, Coetzee N, Zwarenstein M, Lombard C, Guttmacher $\mathrm{S}$, Oxman A, et al. A systematic review of strategies for partner notification for sexually transmitted diseases, including HIV/AIDS. Int J STD AIDS. 2002 May;13(5):285-300.

9. Landis SE, Schoenbach VJ, Weber DJ, Mittal M, Krishan B, Lewis $\mathrm{K}$, et al. Results of a randomized trial of partner notification in cases of HIV infection in North Carolina. N Engl J Med. 1992 Jan 9;326(2):1016.

10. Brown LB, Miller WC, Kamanga G, Nyirenda N, Mmodzi P, Pettifor A. HIV partner notification is effective and feasible in subSaharan Africa: opportunities for HIV treatment and prevention. J Acquir Immune Defic Syndr. 2011 Apr 15;56(5):437-42.

11. Henley C, Forgwei G, Welty T, Golden M, Adimora A, Shields R, et al. Scale-up and case-finding effectiveness of an HIV partner services program in Cameroon: an innovative HIV prevention intervention for developing countries. Sex Transm Dis. 2013 Dec;40(12):909-14. doi: 10.1097/OLQ.0000000000000032.

12. Rutstein SE, Brown LB, Biddle AK, Wheeler SB, Kamanga G, Mmodzi P, et al. Cost-effectiveness of provider-based HIV partner notification in urban Malawi. Health Policy Plan. 2014 Jan;29(1):11526. doi: 10.1093/heapol/czs140.

13. Brown LB, Miller WC, Kamanga G, Kaufman JS, Pettifor A, Dominik RC, et al. Predicting partner HIV testing and counseling following a partner notification intervention. AIDS Behav. 2012 Jul;16(5):1148-55. doi: 10.1007/s10461-011-0094-9.

14. Consultation on Partner Notification for Preventing HIV Transmission. Consensus statement from Consultation on Partner Notification for Preventing HIV Transmission, Geneva, 11-13 January 1989 [Internet]. Geneva: World Health Organization; 1989 [cited 2014 Dec 10]. Available from: http://apps.who.int/iris/handle/10665/61777.

15. Pettifor A, MacPhail C, Corneli A, Sibeko J, Kamanga G, Rosenberg N, et al.; NIAID Center for HIV/AIDS Vaccine Immunology. Continued high risk sexual behavior following diagnosis with acute HIV infection in South Africa and Malawi: implications for prevention. AIDS Behav. 2011 Aug;15(6):1243-50. doi: 10.1007/s10461-010-9839-0.

16. Nuwaha F, Kambugu F, Nsubuga PS. Factors influencing sexual partner referral for sexually transmitted diseases in Uganda. Sex Transm Dis. 1999 Sep;26(8):483-9.

17. Winfield J, Latif AS. Tracing contacts of persons with sexually transmitted diseases in a developing country. Sex Transm Dis. 1985 Jan-Mar;12(1):5-7. 